\title{
Pratiques
}

Linguistique, littérature, didactique

\section{Le déjà-là chez des scripteurs apprenants : des ressources diversement exploitées}

The "already there" amongst novice scriptwriters: some variously exploited resources

\section{Kathy Similowski}

\section{OpenEdition}

\section{Journals}

Édition électronique

URL : http://journals.openedition.org/pratiques/3358

DOI : 10.4000/pratiques.3358

ISSN : 2425-2042

Éditeur

Centre de recherche sur les médiations (CREM)

\section{Référence électronique}

Kathy Similowski, « Le déjà-là chez des scripteurs apprenants : des ressources diversement exploitées », Pratiques [En ligne], 173-174 | 2017, mis en ligne le 10 mars 2017, consulté le 20 avril 2019. URL http://journals.openedition.org/pratiques/3358 ; DOI : 10.4000/pratiques.3358

Ce document a été généré automatiquement le 20 avril 2019.

(c) Tous droits réservés 


\section{Le déjà-là chez des scripteurs apprenants : des ressources diversement exploitées}

The "already there" amongst novice scriptwriters: some variously exploited resources

Kathy Similowski

1 Cet article se fonde sur l'idée apparemment simple selon laquelle l'écriture résulte d'agencements singuliers d'éléments empruntés. En effet, il a été mis en évidence que la production verbale nait en partie du réagencement et de la transformation d'un substrat linguistique préexistant. En sélectionnant des matériaux discursifs déjà présents, en les interprétant, en les agençant, tout écrivain et plus généralement tout scripteur fait acte de création. Aussi la notion de déjà-là utilisée par les linguistes permet-elle de revisiter une problématique ancienne concernant l'écriture, celle des rapports entre invention et imitation.

Dans l'univers scolaire, cette question s'est toujours posée avec une certaine acuité car on a longtemps postulé que la lecture devait précéder l'écriture, les textes littéraires se faisant les pourvoyeurs de mots et d'idées allégeant ainsi la tâche du jeune scripteur, mais bridant du même coup leurs capacités d'innovation, les pédagogues du XIX siècle y voyant même une volonté de "discipliner l'imagination" enfantine (Jey, 2006, p. 29). L'histoire de l'enseignement de la «composition française " nous montre en effet comment la littérature a servi de modèle esthétique et moral à des générations d'élèves (Boutan, 1996, p. 221-222; Chervel, 2008, p. 707) avant qu'une plus grande liberté et expressivité du sujet scripteur ne soit recherchée à travers des écrits "authentiques » (Bishop, 2006, p. 29; 2007, p. 23). Ne se contentant pas de ce traditionnel postulat transmissif, les didacticiens ont examiné comment pouvait fonctionner l'articulation entre la lecture et l'écriture. L'intérêt pédagogique des écritures par imitation a ainsi été étudié de près (Petitjean, 1984). Plus récemment, l'inscription en 2002 de la "littérature " dans les programmes de l'école primaire a permis d'ancrer le travail d'écriture sur des 
textes du patrimoine ou d'auteurs de littérature de jeunesse fournissant une partie du matériau imaginaire mais aussi un lexique et des caractéristiques stylistiques qui peuvent servir de socle à l'écriture des élèves (Branca-Rosoff \& Garcia-Debanc, 2007, p. 47). Ce retour officiel de la littérature a été également l'occasion de réinterroger les notions de fiction (Plane, 2006b) et de création (Doquet-Lacoste, Lumbroso \& Tauvernon, 2009, p. 7 ; Boré \& Calil, 2013, p. 12). On a notamment mis à l'épreuve des dispositifs didactiques ayant pour finalité de conduire les jeunes scripteurs à puiser dans leurs lectures la matière de leur écriture. Ainsi, la mise à disposition de textes ressources favoriserait au cours d'une séquence d'écriture des ajouts significatifs touchant à la macrostructure des textes et développerait in fine " une meilleure compétence » des élèves «à étoffer leurs récits " (Crinon, 2006, p. 66). Des études se sont attachées à repérer dans les écrits de collégiens les réminiscences de lectures antérieures (Fradet, 2006). D'autres se sont intéressées aux différentes manières dont les élèves mettent en mémoire à la fois un texte source entendu et leur propre texte (Plane, Rondelli \& Vénérin, 2013).

vrai que tous les élèves/auteurs s'inspirent de textes lus, entendus ou écrits (François, 1990, p. 183), il n'en demeure pas moins que la manière dont ils le font, diffère d'un élève/auteur à l'autre. L'article explorera cette question en s'appuyant sur une expérimentation dont les analyses sont actuellement en cours et qui cherche à explorer le déjà-là dans l'écriture scolaire. Plus précisément, il s'agit de décrire la manière dont des scripteurs débutants emploient et réinvestissent des matériaux linguistiques lors d'une réécriture.

4 Après avoir posé les contours théoriques de nos observations et présenté notre dispositif, nous tenterons de catégoriser les formes d'emprunt et de mettre en évidence des fonctionnements idiosyncrasiques qui témoignent des différentes façons d'être auteur de son texte.

\section{Des notions en débat}

5 Le propos de cet article étant de s'interroger sur les effets du déjà-là chez de jeunes scripteurs au sein d'une séquence d'écriture scolaire intégrant une réécriture, il convient à titre liminaire de préciser dans quelle acception ces notions seront entendues. Par ailleurs, nous évoquerons les termes d'emprunt et de reformulation nous permettant de caractériser les procédés de captation et d'absorption des matériaux linguistiques utilisés par les élèves.

\subsection{Le déjà-là comme réservoir de l'écriture}

Dans le champ littéraire et depuis longtemps les textes sont examinés sous l'angle de l'imitation et de l'invention. Or le déjà-là est invoqué comme relevant à la fois de l'une et l'autre de ces notions. S. Plane (2006a, p. 3) nous rappelle que pour certains écrivains "l'écriture est animée par la quête d'un déjà-là qui sans cesse se dérobe ». Pour B. Daunay (2004, p. 29) la notion même d'invention empruntée à la rhétorique doit être conçue comme inhérente à la reprise d'un texte source. Il cite à ce sujet R. Barthes (1970, p. 198) : "l'invention renvoie moins à une invention (des arguments) qu'à une découverte: tout existe déjà, il faut seulement le retrouver: c'est une notion plus "extractive" que “créative" » et conclut: «Le travail d'invention est le repérage et la manipulation d'un matériau déjà-là.» 
7 Pour les didacticiens, écrire une fiction dans le milieu scolaire appelle à se souvenir de « sédiments (stéréotypes, scripts, scènes, motifs, personnages, bribes de phrases) laissés par des histoires entendues, lues, déjà imaginées » (Tauveron \& Sève, 2005, p. 28). Il s'agit donc d'encourager les élèves à intégrer leurs expériences et leur mémoire de lecture à la pâte de leurs textes, à réemployer telle formule rencontrée. Pour J.-L. Dufays et B. Kervyn (2004, p. 120), il faut « dire clairement que ce qu'on attend (des élèves), c'est moins un travail d'invention au sens moderne du terme qu'un travail d'inventio, c'est-à-dire d'exploitation et de modalisation de ressources langagières déjà-là, qui relèvent largement de la stéréotypie ». Ces points de vue bouleversent en partie les conceptions scolaires classiques de la production. En effet, D. Dormoy (2004, p. 57-58) fait remarquer que si toute écriture est un réarrangement, alors toute invitation scolaire à l'écriture devrait s'accompagner de la mise à disposition d'un corpus textuel dans lequel les élèves vont pouvoir se nourrir. C'est d'ailleurs « un travail de cut-up et de réassemblage » plus difficile qu'il n'y parait, car il conduit «à se poser des questions sur la succession des éléments et surtout sur l'essentiel travail de choix parmi de nombreux possibles ».

8 C'est cette activité de réécriture-réorganisation de textes déjà-là qui nous intéresse. Nous choisissons de nommer déjà-là le substrat présent lors de l'activité d'écriture dans ses aspects linguistiques et diégétiques qui se glissent dans la plume de l'élève lorsqu'il écrit ou réécrit. Ce qui nous conduit à envisager la notion de réécriture.

\subsection{La réécriture}

9 Le numéro 115/116 de Pratiques, "La réécriture », montre comment cette notion est entrée dans les différentes strates du système éducatif et a été diversement définie. Le terme a connu une vaste diffusion notamment en littérature pédagogique. D. Bessonnat (2000, p. 5-7) le distingue de notions connexes comme la récriture (« venant après-coup comme une réorientation »), la correction (confronter le texte à une norme) et la révision (laquelle dans le modèle des processus rédactionnels comprend une lecture critique et une mise au point incluant correction et réécriture). Selon C. Boré (2000; Boré \& Huynh, 2004, p. 3) on peut globalement retenir deux acceptions qui prennent l'une et l'autre leur source dans le principe dialogique de $\mathrm{M}$. Bakhtine. La première qui s'apparente à l'intertextualité, «c'est-à-dire à l'idée que l'autre (comme individu, comme collectivité, comme texte) est toujours déjà-là dans le discours " et se fonde sur les travaux de J. Kristeva (1969) et ceux de G. Genette (1982). La seconde entendue comme «le retour d'un sujet sur son propre écrit » et qui renvoie notamment à la critique génétique et aux travaux de l'ITEM ${ }^{1}$.

10 Le cadre théorique de notre travail s'inscrit dans cette référence au dialogisme et à l'intertextualité comme dans celle de la critique génétique.

11 En ce qui concerne la tâche des élèves dans notre protocole, le terme recouvre plusieurs réalités :

- l'écriture d'une suite de texte qui pourrait être analysée en une hétéro-réécriture (Plane, 2000, p. 2) parce que réorientant la lecture d'un texte enclencheur, écrit par un autre (i.e. un écrivain);

- une nouvelle écriture de texte ou une auto-réécriture (ibid.) dans un contexte particulier puisque cette opération s'est effectuée sans qu'il y ait eu coprésence des deux textes, ce qui fait que le lien entre le texte premier et le texte second peut être distendu et ainsi interroger la notion de réécriture. 
mécanismes de fabrication par emprunts et juge les textes produits « intéressants »: « Là chacun crée en reprenant-modifiant certains éléments du texte " et cela avec une «compétence textuelle» qui fait que les changements de temps, de lieux, de genre, obéissent à une «logique textuelle » inconsciente. Nous avons aussi été confrontée à ce bouillonnement créatif et nous nous sommes heurtée à un problème de dénomination d'autant plus épineux qu'une terminologie foisonnante issue de la diversification des points de vue disciplinaires existe déjà : paraphrase ${ }^{2}$ qui reste au plus près des mots, des locutions et des constructions, "entendue par Grésillon comme un sous-type de réécriture qui a la propriété de relier A à B dans une grande proximité-sémantique » (Boré \& Doquet-Lacoste, 2004, p. 11), paraphrase imitative ${ }^{3}$, reformulation, notion qui « recouvre un large champ d'opérations métalinguistiques et métadiscursives au point qu'elle ne peut être enfermée dans une définition univoque » (Le Goff, 2006, p. 138), reformulation paraphrastique et non paraphrastique ${ }^{4}$, activité "réitérative » ou «reformulante» (Kara, 2007) mais aussi reprise, réemploi, réappropriation, réagencement, modification, transformation...

18 Nous utiliserons le terme de reformulation avec le sens que lui donne C. Boré (2000, p. 28) c'est-à-dire d'une « reproduction personnelle » d'un discours-source.

Reformuler c'est toujours " prendre place », «s'inventer » l'espace discursif ou habiter plus ou moins le déjà-dit, s'orienter vers le à-dire (Donahue, 2007, p. 247). C'est cet espace d'appropriation du déjà-là que nous avons tenté de disséquer en empruntant aux outils descriptifs déjà investis, conscient que nous travaillons sur des traces sujettes par définition à interprétations, que la variété des procédés échappe à une catégorisation étanche et qu'un scripteur au sein d'un même écrit peut utiliser pléthore de procédés. 


\section{Un dispositif pour observer les traces du déjà-là}

20 caractériser les modes de récupération du déjà-là dans l'écriture des élèves de fin d'école primaire afin d'aider à la mise en place de situations d'enseignement appropriées. Nous souhaitons mettre au jour la nature des matériaux récupérés : ceux que les élèves ont eux-mêmes déjà produits dans une première version et ceux fournis par des textes littéraires, examiner ainsi le rapport entre ces deux sources de substrat que sont le déjàlà de soi et le déjà-là littéraire. L'autre objectif est d'identifier les modes de récupération de ces éléments ainsi que les procédures employées par les élèves pour les intégrer au texte nouvellement produit.

\subsection{Choix méthodologiques}

21 L'objectif expérimental étant d'observer les effets de textes littéraires sur la production écrite, quatre groupes ont été constitués en fonction de plusieurs variables, permettant ainsi la confrontation de données: présence ou non de texte enclencheur, mise à disposition ou non d'un corpus de textes littéraires, nombre de phases d'écriture.

Entre avril et mai 2013, quatre classes de la région parisienne (97 élèves de CM1-CM2 ; milieu social mixte, plutôt favorisé) se sont donc prêtées à la rédaction d'un récit de fiction, écrit particulièrement travaillé en fin d'école élémentaire.

Nous rendrons compte ici des résultats des deux premiers groupes (48 élèves de CM2, âgés en moyenne de 11 ans), la comparaison des productions réalisées par ces deux groupes nous informant sur l'impact d'un texte enclencheur lors d'une première phase d'écriture.

La première phase a consisté à faire rédiger un récit (V1) dans des situations contrastées. Le premier groupe d'écoliers (G1) a reçu uniquement une consigne d'écriture lui enjoignant de produire un récit : «Un enfant arrive sur une ile à la suite d'un naufrage. Raconte. » Le second groupe a reçu un texte d'auteur assorti de la consigne d'en écrire la suite. avec la suite du dispositif.

La seconde phase d'écriture (V2) a eu lieu une semaine plus tard. Les élèves des deux groupes ont reçu un ensemble de quatre textes littéraires, mis à leur disposition sans étayage pédagogique, assorti d'une consigne demandant de produire un texte. Cette consigne n'imposait pas d'utiliser les textes d'auteurs; le dispositif offrait donc aux jeunes scripteurs des ressources qu'ils étaient libres d'exploiter ou non 5 .

27 Nous avions formulé deux hypothèses :

- la présence d'un texte enclencheur en V1 (G2) guiderait les choix des scripteurs, ce qui pourrait être évalué par une comparaison des deux groupes (G1 vs G2),

- la mise à disposition d'un corpus en V2 modifierait les choix des scripteurs, ce qui pourrait être évalué par une comparaison des deux versions (V1 vs V2). Ces apports (linguistiques et diégétiques) pourraient avoir un effet sur la longueur des textes : V2> V1.

Nous avons choisi le genre de la robinsonnade, genre caractérisé par un certain nombre de valeurs et de stéréotypes (Halté, 2004) et qui peut être défini comme «un roman 
d'aventure et d'éducation au cours duquel un enfant est amené à faire naufrage, à être confronté aux problèmes de survie et d'isolement dans une ile coupée de la civilisation d'origine et généralement retrouvé à l'issue de ce temps de retraite initiatique » (DuboisMarcoin, 1997, p. 5).

Nous avons sélectionné des extraits d'ouvrages de littérature de jeunesse, en fonction des critères suivants : longueur (les textes doivent être à la fois consistants et rapidement lus, d'où des extraits allant de 230 à 888 mots), propriétés lexicales (présence de termes peu fréquents mais aisément compréhensibles), instants dans le récit (naufrage, installation sur l'ile), séquences textuelles considérées comme des foyers axiologiques forts (maitrise du feu, quête de la nourriture, construction de l'habitat).

Pour la première phase, le groupe 2, chargé d'écrire une suite de récit, disposait d'un extrait du livre Le royaume de Kensuké relatant le naufrage d'un garçon, Michaël, et de sa chienne, Stella. Le récit se termine sur une exploration de l'ile et le constat de la solitude.

Pour la seconde phase, les deux groupes avaient à leur disposition quatre extraits de robinsonnades situant l'action après le naufrage. Le texte A tiré de L'ile d'Abel évoque la maitrise du feu : Abel retrouve les méthodes ancestrales, émet des signaux de fumée, cuit des graines et des légumes, confectionne de la vaisselle en argile et confie un message au courant. Le texte B est issu du livre Sa Majesté les Mouches. Un avion s'écrase sur une ile déserte. Seuls rescapés et livrés à eux-mêmes, des garçons tentent de s'organiser. L'auteur dépeint une forêt luxuriante où la nature et ses fruits mûrs s'offrent à profusion. L'extrait choisi a fait l'objet de deux modifications pour assurer l'homogénéité du corpus de texte remis aux élèves: suppression des personnages annexes et changement énonciatif (passage à la première personne). Le texte $C$ tiré d'une réécriture pour jeune public de Robinson Crusoé résulte de la combinaison de deux extraits portant sur la quête de la nourriture. Le thème de la construction de l'habitat est introduit par le texte $\mathrm{D}$; sous le titre Vendredi ou la vie sauvage, M. Tournier offre une adaptation pour la jeunesse de son livre Vendredi ou les Limbes du Pacifique. Ces cinq textes ont donc constitué le substrat littéraire fourni.

\subsection{Les données collectées}

Les données recueillies proviennent de 96 copies ( 24 copies pour chaque version et chaque groupe). Il a été procédé à un relevé manuel des formes, complété par une analyse lexicométrique des textes littéraires et des productions d'élèves. Ont été observés les matériaux récupérés du texte enclencheur (groupe 2), des textes sources, des textes déjà produits en première version, et également résultant de souvenirs de lectures antérieures. Une fiche rassemblant tous ces éléments a été établie pour chaque élève. En parallèle, un classement de ces éléments a été opéré afin de déterminer les faits les plus saillants.

\section{Traits caractérisant l'ensemble des textes du corpus}

Nous notons ici les éléments qui permettent d'avoir une image de l'ensemble de notre corpus, afin de faire apparaitre par contraste, ultérieurement, des traits spécifiques à des scripteurs qui contribuent à définir leur profil. 


\subsection{Des textes relativement consistants}

\subsubsection{Des textes pas si courts}

Selon J. Crinon (2006, p. 67) les textes des scripteurs novices sont souvent courts car les apprenants peinent à « déplier la macrostructure en propositions microstructurelles ", c'est-à-dire à donner au lecteur suffisamment d'éléments à voir et à imaginer, à laisser le temps d'une représentation de ce qui lui est offert. Dans notre corpus, certains textes semblent effectivement construits comme une trame, juxtaposant les actions en une liste imprécise ou non développée, mais il existe une très grande variabilité en terme de longueur des textes produits, sachant que la consigne n'imposait rien de ce point de vue.

Ainsi, le nombre de mots oscille (pour les deux versions) entre 119 et 807 avec une moyenne de 327 mots (médiane: 314 ). Si l'on considère que les programmes de 2008 préconisent la rédaction de textes représentant deux paragraphes ou une dizaine de lignes, soit environ 100 à 200 mots, on peut dire que les textes recueillis correspondent à une norme scolaire implicitement attendue.

6 Nous nous attendions à ce que le texte issu de la seconde écriture soit plus long. D'une part, bien que les deux passations soient séparées par un intervalle d'une semaine, la tâche de textualisation semblait plus aisée car elle avait été effectuée une première fois. Il a en effet été mis en évidence qu'un texte continue à " travailler son auteur » (Lejeune, 1995) au-delà de la scription et c'est ce "ressassement» du texte déjà produit qui pourrait expliquer qu'une nouvelle écriture en rappel différé d'un conte écouté conduise à produire des textes plus longs qu'en rappel immédiat (Plane, à paraitre). D'autre part, les élèves disposaient de matériaux linguistiques fournis par les textes littéraires pouvant nourrir leur écriture.

Par ailleurs, nous nous interrogions sur l'impact du texte enclencheur (G2) en considérant qu'il pouvait faciliter lui aussi la tâche des scripteurs en prédéfinissant un scénario et en apportant des matériaux qui pourraient être réutilisés.

L'analyse statistique des données ${ }^{6}$ soumises à des tests de comparaisons de leurs médianes et rangs $^{7}$ fait apparaitre qu'il n'existe pas de différence significative de longueur entre les versions 1 et $2^{8}$. La mise à disposition de textes ressources n'a donc pas eu d'influence sur la longueur des textes. En revanche, il existe une différence significative entre les deux groupes en $\mathrm{V} 1^{9}$. On peut y voir l'impact du texte enclencheur qui trace un cadre programmatique tout en permettant aux élèves d'exercer leur imagination dans la suite de l'histoire, ce jeu de contraintes-liberté les motivant à s'engager plus avant dans l'écriture. Cependant, il n'est pas possible de déterminer si ce facteur est seul responsable de la différence constatée. L'intervention expérimentale (mise à disposition d'un texte enclencheur pour le groupe G2) fait partie des facteurs pouvant avoir contribué à la différence, mais d'autres facteurs peuvent entrer en ligne de compte. On note qu'après répétition, la différence entre les groupes (G2V2 vs. G1V2) existe toujours numériquement, mais perd sa significativité statistique.

Dans notre étude, nous ne pouvons donc conclure à un effet des textes littéraires sur la longueur des textes des élèves. 


\subsubsection{Des textes complets}

Aucune consigne donnée aux élèves ne portait sur le contenu diégétique et aucune indication n'était apportée par les textes littéraires sur la clôture du récit. Or, dans la majorité des écrits, on note la présence d'un dénouement (décision d'installation définitive, départ, mort, réveil, fin d'un tournage...) à l'issue d'une série d'actions et d'un ou plusieurs rebondissements (rencontre, nouveau naufrage, mise en danger du héros...). Les productions écrites sont donc majoritairement des textes complets.

\subsection{Des textes qui puisent un déjà-là à trois sources}

L'examen des productions révèle l'existence d'un déjà-là multiforme provenant de trois sources :

- Des éléments mémorisés avant l'expérimentation (lectures, films, jeux vidéo, réception de discours, productions personnelles) issus des expériences langagières antérieures au protocole. Ces éléments constituent un matériau hétérogène, stocké, mais inégalement consistant et mobilisable au cours des deux versions. On en identifie avec certitude la trace par exemple lorsqu'apparait la référence non équivoque à une lecture antérieure, comme le nom du personnage "Vendredi », qui ne figure pas dans les textes remis aux élèves si ce n'est sous la forme de la référence de l'un d'entre eux (texte D).

- Des éléments issus du texte produit en V1. Nous nous attendions à ce que le texte déjà produit dans la première phase de l'expérimentation soit réinvesti en $\mathrm{V} 2$, mais nous nous interrogions sur le poids qu'il aurait parmi les autres matériaux composant les ressources à disposition du scripteur, attendu que ce texte n'était plus consultable et avait donc des chances d'être flou ou déformé.

- Des éléments issus des textes littéraires fournis aux élèves. Nous pensions également que les textes littéraires fournis apporteraient des éléments programmatiques orientant et facilitant la réécriture, se substituant au déjà-là propre à chaque scripteur.

Nous nous intéresserons ici aux traces, parfois ténues, parfois consistantes, du déjà-là en limitant nos observations aux emprunts de matériaux linguistiques issus du texte déjàproduit et des textes littéraires en V2, et à la comparaison des deux groupes de scripteurs en mettant au jour les reprises du texte enclencheur pour ceux qui en disposaient.

\subsection{Deux modes à l'œuvre pour opérer des emprunts aux textes sources}

Les emprunts sont d'une grande diversité. Pour en témoigner, nous avons choisi quelques exemples tirés des productions en V2. Nous mettrons à chaque fois en regard les textes sources désignés par A, B, C ou D, et les textes cibles identifiés par le prénom de l'élève. L'orthographe des textes d'élèves est conservée.

Deux grands modes sont repérables :

- une fidélité à la forme, aux « mots » du texte, d'une part ;

- une fidélité au sens, ou équivalence sémantique, d'autre part, allant d'une simple restitution à une réinterprétation du texte source. 


\subsubsection{Fidélité à la forme}

La fidélité à la forme se manifeste par le biais de quatre procédés :

- La répétition, avec modalisation ou non, d'un lexème dont on peut affirmer qu'elle correspond à une sélection par l'élève, comme, par exemple, l'emploi du mot « fortification » mot d'usage peu courant et dont on ne trouve d'ailleurs qu'une occurrence dans les textes littéraires fournis aux élèves : je fis des fortification tout autour de l'or et de la grotte. (Geoffroy)

- La reprise totale ou partielle d'un segment. C. Martinot (2007, p. 184) qualifie de répétition lacunaire la situation dans laquelle l'enfant répète à l'identique une partie seulement de l'énoncé source en produisant un énoncé reformulé qui peut être agrammatical et/ou lacunaire du point de vue des informations à restituer.

Texte C : j'y trouvai trois chevreaux, un mâle et deux femelles.

Alors j'ai pris les trois chevreaux et une femelles que j'ai relâché pour qu'ils se reproduisent. (Quentin)

Texte A : J'utilisai d'abord mes feux pour envoyer des signaux de fumée, destinés à attirer l'attention de quelque créature civilisée, éventuellement présente, au-delà du cours d'eau.

J'utilisai d'abord mes feu pour envoyer des nuages de fumée pour attirer l'attention. (Nina)

- Le tissage par assemblage. Nous nous proposons d'appeler ainsi la récupération de mots ou de segments d'un ou plusieurs textes et leur intégration dans une même phrase :

Texte A : Quelques jours plus tard, je réussis à allumer un feu.

Texte B : Je marchais comme un habitué.

Quelque jour plus tard je m'habite a cette vie. (Enzo)

- Le tissage par réagencement. Des éléments issus d'un même texte source peuvent être réorganisés et changer de place lors de la linéarisation du message

Texte $\mathrm{D}$ : Je situai ma maison près d'un grand cèdre au centre de l'île. Je creusai d'abord un fossé rectangulaire que je meublai d'un lit de galets recouverts euxmêmes d'une couche de sable blanc. Sur ces fondements parfaitement secs et perméables, j'élevai des murs en mettant l'un sur l'autre des troncs de palmiers. La toiture se composa d'une vannerie de roseaux, sur laquelle je disposai ensuite des feuilles de figuier-caoutchouc en écailles...

C'est au moment d'entrée que je decida de faire une maison. Je rassembla tout ce qui pouvait me servir: tron de palmiers, galets, sable, feuilles de figuierscaoutchou... Je commeça par creuser une fosse rectangulaire je mis des galets tout au fond et recouvris de sable, je fis de même une seconde fois. Je fis les murs en trons de palmiers... (Pierre-Yves)

\subsubsection{Fidélité au sens}

Selon C. Martinot (2007, p. 201), c'est à 10 ans que les reformulations par équivalence sémantique sont les plus nombreuses et les restructurations les plus complexes. Parce qu'il s'agit ici non pas de reformulation dans un contexte d'acquisition du langage dans lequel l'enfant cherche plus à reproduire qu'à transformer mais de production écrite laissant le scripteur libre de ses choix, nous interprèterons plus souplement certaines des catégories proposées par cette auteure. On notera la proximité de ces procédés avec ceux mis en œuvre dans le fonctionnement anaphorique, ce qui signale que des mécanismes de mémorisation similaires sont mobilisés.

Ce type d'emprunt qui privilégie la fidélité au sens se manifeste sous les trois formes suivantes : 
(Martinot, 2007, p. 183) :

Texte A: Plus tard, je fis cuire divers légumes... dans des plats faits d'une argile rougeâtre que j'avais trouvée vers le sud de l'île.

En fin d'après midi dans le sud de l'île, il trouva de l'argile rouge puis il en fit des plat. (Basile)

2) La paraphrase sémantique, sans contrainte sur la structure ou le lexique (ibid.), qui comprend elle-même plusieurs variantes :

a) La paraphrase résomptive ou résumante lorsque l'information du texte source est synthétisée :

Texte A : J'utilisai d'abord mes feux pour envoyer des signaux de fumée, destinés à attirer l'attention de quelque créature civilisée, éventuellement présente, au-delà $\mathrm{du}$ cours d'eau. Mon feu allumé, je le recouvrais en partie de feuilles humides et bientôt s'en élevait une épaisse fumée blanche.

je commença à envoyer des singnaux et je mis des feuilles humides pour faire de la fumer. (Mahery)

51 b) La paraphrase expansive, lorsque, à l'inverse, le scripteur procède à une extension à partir d'un élément du texte source :

Texte C : il m'arriva de trouver... un petit sac qui avait été empli de grains

Il y trouve un sac remplit de graines de maïs, un peu de clous, un marteau.

(Raphaël)

52 c) La paraphrase contextualisante ou recontextualisante, lorsque l'élève prend ses distances avec le texte source et propose une (autre) cause, un (autre) but, des (ou d'autres) conséquences pour expliquer les actions de son récit :

Texte A : Quelques jours plus tard, je réussis à allumer

$\mathrm{Au}$ bout d'un moi l'hiver surgi et Michael se rapela de son cour d'histoire sur les

hommes de la préhistoire pour faire du feu pour se réchaufer et pour faire cuire des aliments. (Victor)

d) La paraphrase explicative qui ajoute une information destinée à rendre plus compréhensible les éléments repris. Dans l'exemple suivant l'écolière y recourt en présence de ce qui lui parait être une ellipse narrative :

Texte A : Quelques jours plus tard, je réussis à allumer un feu

Quelques jours plus tard, j'ai esaillé de faire du feu et j'ai réussi. (Samira)

54 e) La reformulation interprétative qui consiste à apporter un jugement. Ainsi dans l'exemple qui suit, il s'agit de caractériser le mot «jungle » en montrant qu'il s'agit d'un univers potentiellement dangereux :

Texte B : Je me trouvai bientôt en pleine jungle...

Dans la jungle il y avait peut-être des serpents venimeux, des tigres, des lions, mais aussi pleins de merveille si je sortais de cette jungle par exemple des éléphants, la vallée de Tembo. (J'avais lu ça dans un livre) Oui elle était magnifique cet vallée. (Thaïs)

\section{3) La substitution de lexème}

On relève plusieurs types de substitutions :

a) La relexicalisation ou la synonymie lorsque les matériaux proposés ne paraissent pas adaptés au jeune scripteur. C'est le cas de Judith qui explique lors d'un entretien qu'elle a préféré le mot « cabane » au mot maison (fourni par le texte D), compte tenu du fait que la construction s'opérait sur une ile. Ce procédé peut aussi être dû au fait que les mots du 
texte n'appartiennent probablement pas à la mémoire sémantique de l'élève, ce qui l'amène à puiser dans un répertoire plus proche :

Texte $\mathrm{C}$ : des épis d'une orge verte et parfaite une plantation de blé (Maximin)

b) L'hyponymie, qui permet d'apporter plus de précision :

Texte D : quelques meubles en osier

je me construis une chaise en osiers (Pierre-Yves)

c) L'hyperonymie, qui exerce une fonction résumante

Texte A : des plats faits d'une argile rougeâtre

Il fit de meubles et de la vaisail en argil (Nasrine)

Ces exemples de fidélité formelle et d'équivalence sémantique attestent la diversité des procédés utilisés par les élèves pour créer un récit à partir d'un stock linguistique déjà-là.

\subsection{Les effets de l'emprunt}

61 L'insertion partielle de matériaux en début de texte peut servir de point d'appui à une narration plus personnelle. Ainsi, dans les incipits s'observent des reprises de segments des textes littéraires ou même de la consigne d'écriture. C'est le cas d'Édouard qui commence son texte par: "Un enfant arrive sur une île à la suite d'un naufrage ». Disséminées dans la réécriture, les informations issues des textes sources peuvent contribuer à ce que D. Bucheton (1996) appelle un épaississement du texte car la réécriture, alors que l'élève n'a pas son premier écrit sous les yeux, permet à « la penséelangage de se remettre en mouvement » puisque le scripteur ne cherche pas à corriger ou à améliorer le texte déjà produit mais prend de la distance, récupère pour inventer (Bucheton et al., 2000). De même, J. Crinon (2006, p. 68) constate que ce qui permet « de donner de l'épaisseur aux récits c'est l'importance directe d'expressions tirées des textes modèles, points de départ à des expansions donnant à voir et à entendre la scène ». Les textes ressources contribuent à cet enrichissement de deux manières ; ils constituent des réservoirs de mots et d'idées qui peuvent libérer certains apprenants de leur sentiment d'être impuissants à inventer, et ils permettent de convoquer d'autres mots et idées qui font déjà partie du patrimoine culturel de l'enfant.

Cependant, ces emprunts peuvent générer aussi des tensions car le jeune scripteur doit intégrer la matière première littéraire à sa propre pâte textuelle. Ce faisant, il sélectionne des matériaux qu'il réinterprète, parfois de façon erronée. Ainsi, Pierre-Lou préfère au terme obsolète "haut-de-chausse » une synonymie erronée, en habillant son héros de « chaussettes». Basile ne comprend pas qu'un « lit de galets » sert aux fondations de la maison du naufragé, si bien qu'il fait dormir son personnage sur un « lit de galets ». Il y a ici déformation du substrat initial. Toutefois comme le dit J. Crinon (2006, p. 69) il arrive "qu'un faux sens soit heureux». Le personnage d'Eloi nous dit: «chaque jour... (je) civilisais mon habitat ». On peut penser que l'élève se réfère à Robinson essayant de créer une " atmosphère confortable et intime » (texte $\mathrm{D}$ ) et d'" attirer l'attention de quelque créature civilisée » (texte A). L'expression retenue est peut-être critiquable mais elle est originale. L'insertion par empan important ou par placage sans appropriation peut conduire également à des problèmes de cohésion car la continuité thème/rhème est rompue. L'intelligibilité même du texte peut en être affectée : 
Tableau 1. Texte de Ernestine en version 2 comparé au texte A

\begin{tabular}{|c|c|}
\hline Texte A & Texte d'Ernestine \\
\hline $\begin{array}{l}\text { Quelques jours plus tard, je réussis à allumer } \\
\text { un feu. J'avais entendu parler en classe des } \\
\text { méthodes en usage chez les primitifs, mais } \\
\text { ne m'y étais moi-même jamais essayé...Mes } \\
\text { feux possédaient } \\
\text { la même magie que ceux des ancêtres de la } \\
\text { préhistoire. }\end{array}$ & $\begin{array}{l}\text { Quelques jours plus tard il arriva a allumé un feu. } \\
\text { Depuis qu'il était tout petit, il a pris l'abbitude de } \\
\text { voir comment sa ce passe à la Préhistoire il était } \\
\text { devenu un singe. Alors tua une grosse bête pour } \\
\text { la faire griller au feu. } \\
\text { Car le feu à de la magie... }\end{array}$ \\
\hline
\end{tabular}

Ces intégrations peuvent perturber le schéma initial et provoquer des glissements du « je » au « il » ou inversement, comme chez Quentin :

Après j'ai taillé les arbres pour qu'ils aient de la lumière et pour qu'ils fleurissent et donne des fruits. Puis le lendemain matin il alla continuer de creuser le ruisseau [...] Michael fit du feu pour se réchauffer. Il alla traire les chèvres pour avoir du lait et les mit dans des seaus que j'avais retrouvé sur la plage.

De tels décrochages énonciatifs ont été observés chez 25 \% (6/24) des scripteurs ayant eu à construire un récit avec mise à disposition des textes littéraires (G1V2).

Tous ces dysfonctionnements existent, mais ils sont peut-être la contrepartie du processus d'apprentissage qui est à l'œuvre dans la reformulation. Si l'acquisition du langage « a lieu non pas parce que l'enfant sait imiter ce qu'il entend mais parce qu'il peut transformer ce qu'il entend» (Martinot, 2007, p. 182; 2015, p. 4) alors de même l'apprentissage de l'écrit passe-t-il probablement par la transformation d'un discours source, parce qu'en reformulant l'enfant apprend progressivement à formuler.

\section{Fonctionnements idiosyncrasiques : tendances partagées et singularités}

66 Si les procédés pour s'approprier les éléments fournis sont très divers, en revanche, la manière d'y recourir caractérise les scripteurs. À travers quelques exemples nous montrerons quelles tendances se dégagent et comment les élèves observés maintiennent ou transforment du déjà-là d'une façon qui leur est propre.

\subsection{Variations chez un même scripteur}

67 Le plus souvent, les scripteurs recourent à une diversité de procédés pour emprunter aux textes sources et reformuler. Deux exemples illustreront cette tendance majoritaire.

Ainsi Sabrine reprend un segment du texte $C$ : « Au milieu de tous mes travaux, il m'arriva de trouver, en visitant mes bagages, un petit sac» sous la forme «Au milieu de tous mes travaux il m'arrivai visitant l'île de trouver des bagages et un sac ». Le groupe nominal «un sac» est une reprise partielle de «un petit sac». Le terme "visitant» est recontextualisé : le héros ne visite plus l'ile, il «visite» ses bagages. Sabrine comble les vides du texte $C$, qui décrit le contenu du sac mais non son ouverture, en insérant une paraphrase explicative: «je pris le sac en premier et je l'ouvrit». C'est par une 
paraphrase définitoire qu'elle conclut: «Il n'y avait que des miette de graines » nous livrant sa propre définition de "balle », mot dont le sens dans le texte C était précisé par la note « Balle : enveloppe du grain des céréales. »

De même, lorsque Margaux écrit «La maison d'Alex etait faites d'argiles et de grandes feuilles de bananiers » elle emprunte à plusieurs textes et opère au moyen de plusieurs procédés. Le texte $\mathrm{D}$ développe l'idée d'une construction d'une maison sur la toiture de laquelle sont disposées "des feuilles de figuier-caoutchouc». Le texte A explique la fabrication de plats en argile. Margaux ne choisit pas de mettre son naufragé à l'ouvrage mais montre la maison d'un autre personnage. Elle recontextualise la construction de la maison du texte D et crée ainsi la formule «la maison d'Alex était faite d'argile ». Elle procède par substitution et relexicalisation lorsqu'elle caractérise le matériau "feuilles " en lui adjoignant l'adjectif "grandes» et le syntagme prépositionnel «de bananier». Ainsi s'entrecroisent et se chevauchent plusieurs types de reformulations.

\subsection{Fidélité à une tendance}

70 Si les procédés employés sont divers, en revanche, l'orientation générale peut rester stable tout au long de l'activité d'écriture. Ainsi certaines productions manifestent une tendance à rester fidèle à la forme du texte auquel elles empruntent des éléments, tandis que d'autres recourent de préférence à la reformulation. Deux exemples qui reprennent manifestement des éléments au même texte source témoignent de ces tendances contrastées :

Tableau 2. Textes en version 2 de Pierre-Lou et de Valentine comparés au texte D

\begin{tabular}{|l|l|}
\hline Texte D & $\begin{array}{l}\text { C'est peu après cette première récolte que j'eus la très grande joie de retrouver } \\
\text { Tenn, le chien de La Virginie. L'animal jaillit d'un buisson en gémissant et en } \\
\text { tordant l'échine, me faisant ainsi une vraie fête. }\end{array}$ \\
\hline $\begin{array}{l}\text { Texte de } \\
\text { Pierre- } \\
\text { Lou }\end{array}$ & $\begin{array}{l}\text { Quelle que jours plus tard après cette troisième récolte sur l'île je retrouvait mon } \\
\text { chien Taidi. Le chien s'autère sur son maître et le léchait. }\end{array}$ \\
\hline Texte de & $\begin{array}{l}\text { Soudain un petit bruit se fait entendre. Camille se réjouit, tout espoir n'est pas } \\
\text { perdu! } \\
\text { Valentine se rapproche de l'endroit où il semble provenir. Et elle voit sa chienne ! } \\
\text { Ouaf Ouaf fait le chien. « Oh Diane tu m'as tellement manqué ! »crie Camille. }\end{array}$ \\
\hline
\end{tabular}

71 Comme on le voit, Pierre-Lou privilégie la fidélité formelle : il utilise le calque du texte D en reprenant une partie du lexique initial alors que Valentine recherche plutôt l'équivalence sémantique en s'attachant uniquement au script fourni à partir duquel elle écrit sa propre partition. 


\subsection{Réemploi du déjà-là culturel de préférence aux matériaux fournis par les textes sources}

Certaines productions s'appuient manifestement sur des éléments exogènes, propres à la culture personnelle du scripteur-apprenant. Dans de tels cas, il arrive que le scripteur fasse abstraction des matériaux fournis pour accompagner la production.

\subsubsection{Convocation de matériaux culturels appartenant au même genre que les textes fournis}

73 Certains élèves sont manifestement en mesure de faire appel à une culture littéraire personnelle que la présence des textes sources réactive. C'est le cas d'Enzo qui a eu à produire une suite de récit. Son premier texte est court (172 mots) mais comporte déjà en germe tous les éléments d'un bon récit: une suite d'actions, une complication (« un mirage »), un dénouement. Son récit ressemble à une liste d'évènements non développés : le héros recherche de la nourriture, trouve une chèvre, apprend à parler à un perroquet, construit une ferme, fait du feu, s'habille de fourrure, croit apercevoir un bateau, construit une cabane, ramasse du bois, nourrit ses animaux, est récupéré par un bateau et retourne sur l'ile "pendant les vacances». Le texte manque encore d'épaisseur, mais il s'inscrit pleinement dans l'univers de la robinsonnade.

Le second écrit, plus long (192 mots), maintient le genre. L'élève ne récupère des textes sources que l'incipit mais il convoque ses souvenirs de lectures antérieures: arrivée du «bon sauvage » baptisé confusément «Lundi» et «Vendredi», cannibalisme... Ce sont des marques d'intertextualité extérieures au protocole, mais que les textes ressources ont permis de faire ressurgir :

Quelque jour plus tard je m'habitue a cette vie je ressit à allumer du feu, ma ferme commenca à avoir des animaux des poules du bœuf, des œuf et tout plein d'animaux J'utilisai du feu pour me reperer mes en vain Un jour un bateau arriva des hommes et un homme attacher avec du feu il voulait le manger et je vais vite courir sur mon bateau et je vais chercher mes arme pour sauver cette homme, je tira sur les hommes et j'allais delivre cette homme. Je lui demande pourquoi il voulait le manger mais il comprenez pas. Les jour passa et je lui apris a parler, a s'occuper de la ferme et je lui donna un prenom Lundi comme je l'ai rencontre lundi. Un jour un bateau espagnol vien et dit que ses le père de vendredi et Vendredi dit quil veut rester ici et son pére accepte et repart. Puis un autre jour un bateau anglais vien pour sauver Lundi et Michael qui ont été appelé par les espagnol puis Michael retourne chez lui explique à sa famille ce qui c'est passe et Lundi devient de la famille.

\subsubsection{Convocation de matériaux culturels relevant d'autres genres}

Certains élèves font appel à des éléments manifestement issus de leurs lectures mais n'appartenant pas au genre de la robinsonnade. Le phénomène n'est pas étonnant lorsque les élèves n'ont pas eu à leur disposition le corpus de textes qui fournissait des exemples permettant d'identifier des caractéristiques du genre. Il est plus surprenant lorsqu'il se produit alors que ces matériaux avaient été fournis.

C'est le cas de Clément qui avait à produire une suite de texte et qui, lors de la production de la première version, a bien pris appui sur le texte source qui lui était fourni, mais qui, lors de la réécriture, a convoqué des éléments issus d'autres genres. Ainsi sa première 
version consiste en une suite de récit relativement longue (499 mots) qui procède par reprises et reformulations du texte enclencheur :

Tableau 3. Texte de Clément en version 1 comparé au texte enclencheur

\begin{tabular}{|l|l|}
\hline Texte enclencheur & Texte de Clément \\
\hline $\begin{array}{l}\text { J'avais l'impression d'être épié par des } \\
\text { milliers d'yeux inquisiteurs. }\end{array}$ & $\begin{array}{l}\text { (j'ai) traverser la foret avec toujours } \\
\text { cette impression que des yeux me suivait du } \\
\text { regard à l'entré de la foret }\end{array}$ \\
\hline $\begin{array}{l}\text { Je crois que Stella aussi était inquiète, car } \\
\text { elle restait étrangement silencieuse depuis } \\
\text { que nous avions pénétré dans la forêt. }\end{array}$ & $\begin{array}{l}\text { Stella était très agiter et semblait avoir reconu } \\
\text { une odeur dans le bateau }\end{array}$ \\
\hline $\begin{array}{l}\text { Je scrutai l'horizon. S'il y avait une voile } \\
\text { quelque part sur la mer, je ne pourrais pas } \\
\text { la voir dans cette brume de chaleur. }\end{array}$ & $\begin{array}{l}\text { qlle me montra du museau la mer, j'ai regarder ce } \\
\text { quatre voiles flotantes dans l'air qui pressait à } \\
\text { côté de l'île } \\
\text { à toute vitesse }\end{array}$ \\
\hline
\end{tabular}

77 La réécriture prend ses distances avec ce premier écrit et avec le corpus d'extraits de robinsonnades fourni pour étayer cette réécriture : c'est un tout autre texte que Clément produit, plus court (398 mots), relevant d'un autre genre puisqu'il convoque des figures monstrueuses et mythologiques. La conséquence de cette mise à distance se marque par la quasi disparition des matériaux linguistiques qui avaient été prélevés dans le texte source et réinvesti en V1. Autrement dit, dans ce cas, le déjà-là culturel a fait écran aux ressources fournies lors de l'activité d'écriture.

\subsection{Utilisation de la première version produite comme source de la réécriture au détriment du corpus fourni}

Comme nous l'avons dit plus haut, les élèves avaient à produire un écrit mais cet écrit n'était plus à leur disposition lors de la réécriture. Cependant, pour une partie d'entre eux, cet écrit hors de leur portée, continue d'être présent et s'impose au détriment du corpus de textes qui devait accompagner la réécriture.

Dans les textes produits par Margaux le phénomène se manifeste d'abord par la reprise en V2 du contenu diégétique qui avait été développé en V1. Margaux avait ouvert son premier récit par le monologue de la naufragée :

«Ah!!» « où suis-je? » resonne (une voix) à travers toute l'île. Une fille seule allongée sur la plage vient de se réveiller. La pauvre est terroriser comment est-elle arriver là ? Où sont ses parents? ses amis?

L'héroïne fait la connaissance d'un garçon. Le récit se termine par le réveil de la jeune fille à son domicile parisien : c'était un rêve mais elle doit partir en voyage en bateau dès le lendemain...

81 Le scénario est repris en V2 : 
Le soleil se levait, les vagues percutait le sable blanc et là au millieu de tout ca se trouvait une jeune fille. Elle avait les cheveux chatins, les yeux marron clair et dormait paisiblement. la récupération des matériaux linguistiques employés lors de la production du premier écrit. Margaux procède principalement par autocitations ou autoreformulations, alors que le texte source n'est plus disponible matériellement, il n'est présent que dans la mémoire de Margaux :

Tableau 4. Textes de Margaux en versions 1 et 2

\begin{tabular}{|l|l|}
\hline V1 & V2 \\
\hline Une fille se réveilla en sursotant & $\begin{array}{l}\text { un jeune homme vint la réveiller, } \\
\text { elle sursauta. }\end{array}$ \\
\hline $\begin{array}{l}\text { Pour arriver à côté d'un garçon couché qui } \\
\text { avait l'air de dormir paisiblement. }\end{array}$ & $\begin{array}{l}\text { Elle avait les cheveux chatins, les yeux marron clair } \\
\text { et dormait paisiblement. }\end{array}$ \\
\hline $\begin{array}{l}\text { Amy lui espliqua qu'ils étaient arriver à } \\
\text { (la) suite d'un naufrage. }\end{array}$ & $\begin{array}{l}\text { Elle sappelle Marine, et elle est rescaper d'un } \\
\text { naufrage }\end{array}$ \\
\hline $\begin{array}{l}\text { Elle se lève regarde autour d'elle et voit de } \\
\text { grand arbres tropicaux. }\end{array}$ & $\begin{array}{l}\text { Au village il y avait de toutes [sortes de] choses des } \\
\text { banane, des noix de coco, des fruits tropicaux }\end{array}$ \\
\hline $\begin{array}{l}\text { Amy cuisina les champignons et ils } \\
\text { mangèrent }\end{array}$ & $\begin{array}{l}\text { Ils l'accueillirent et la firent manger à la table } \\
\text { familiale }\end{array}$ \\
\hline Ils s'allongèrent tout deux et dormèrent & Il lui proposa de dormir chez lui \\
\hline
\end{tabular}

Le texte déjà-produit semble verrouiller la capacité re-créatrice du scripteur.

\subsection{Abandon de la première version et récupération des matériaux fournis par le corpus}

À l'inverse du cas précédent, l'apport du corpus fourni pour étayer la production de la deuxième version conduit des élèves à délaisser ce qu'ils avaient eux-mêmes produit pour puiser aux textes sources. Cette réorientation se traduit en tout premier lieu par un changement de système diégétique qui fait que la seconde version est plus conforme aux caractéristiques de la robinsonnade. La reprise d'éléments linguistiques issus du corpus constitue le médium de cette réorientation.

Ainsi Pierre-Lou, qui appartenait au groupe ayant à rédiger un écrit à partir d'une simple consigne, avait composé en V1 un récit (286 mots) mêlant aventure et conte. Son personnage découvre une ruine dans laquelle se trouvent des mages et un monstre emprisonné dans la glace. Natsu échappe à la poursuite du monstre qui disparait dans 
l'eau. L'univers insulaire sert de simple décor à une intrigue qui se déroule sur une journée. La seconde version, quoique plus courte (210 mots), se nourrit des thèmes des textes sources : après une récolte, Éric retrouve son chien, visite l'ile puis construit sa maison et extrait quelques affaires de son navire. Huit ans plus tard, il est repéré par un bateau. Cette deuxième version emprunte directement des éléments aux textes fournis :

Tableau 5. Incipit du texte de Pierre-Lou en version 2 comparé à l'incipit du texte D

\begin{tabular}{|c|c|}
\hline Texte D & Texte de Pierre-Lou \\
\hline $\begin{array}{l}\text { À la fin de l'après-midi du } 29 \text { septembre } 1759 \text {, } \\
\text { Robinson laisse à York sa femme et ses deux } \\
\text { enfants, pour explorer l'Amérique du Sud }\end{array}$ & $\begin{array}{l}\text { À la fin de la matiné du } 11 \text { février 2002, Eric } \\
\text { laisse à Athènes sa femme et son chien pour } \\
\text { explorais les îles du Pacifique }\end{array}$ \\
\hline
\end{tabular}

De la même façon, Nina qui avait eu quant à elle à rédiger une suite de texte en première version, procède à de profonds remaniements une fois en contact avec le corpus. La suite de récit produite par Nina en V1 dépeint un monde fantastique dans lequel Michaël trouve " une serrure dorée d'une petite trappe », et parvient à comprendre le langage de sa chienne « à l'aide d'une sorte d'écouteurs ».

En V2, Nina se livre à un véritable pillage textuel en reprenant les motifs du genre. Ces emprunts ne se font pas sans problème de textualisation, mais le genre est construit :

Tableau 6. Texte de Nina en version 2 comparé au texte D

\begin{tabular}{|l|l|}
\hline Texte D & Texte de Nina \\
\hline $\begin{array}{l}\text { C'est peu après cette première récolte que } \\
\text { j'eus la très grande joie de retrouver Tenn, } \\
\text { le chien de La Virginie. L'animal jaillit d'un } \\
\text { buisson en gémissant et en tordant l'échine, } \\
\text { me faisant ainsi une vraie fête. Je ne sus } \\
\text { jamais comment le chien avait passé tout ce } \\
\text { temps dans l'île, ni pourquoi }\end{array}$ & $\begin{array}{l}\text { grande joie de retrouvé Tenn, le chien de la } \\
\text { gémissant en remuant la queu me fesant une } \\
\text { il n'était pas venu plus tôt à moi. }\end{array}$ \\
$\begin{array}{l}\text { lassi longtemps dans l'île ni pourquoi il n'était } \\
\text { aus venu la plus tôt, mais en_tout cas j'était } \\
\text { content de le revoir. }\end{array}$ \\
\hline
\end{tabular}

Ces deux exemples montrent comment les textes ressources fournis en V2 peuvent redéfinir le cadre du récit et largement déterminer le lexique utilisé.

\subsection{Recours cumulé à des déjà-là}

Certains scripteurs parviennent à conjuguer habilement plusieurs sources d'emprunts.

91 C'est le cas de Thaïs qui produit de longues suites de récits (807 et 577 mots). Le vocabulaire est varié et les motifs du genre sont développés et nourris à la fois par sa culture personnelle, le texte enclencheur (V1), le texte déjà produit et les textes sources (V2). L'élève convoque toutes sortes de déjà-là : 
Tableau 7. Texte de Thaïs en version 2, ses sources et procédés d'emprunts

\begin{tabular}{|c|c|c|}
\hline Sources & Procédés & Thaïs V2 \\
\hline Le déjà-là culturel & $\begin{array}{l}\text { Reprise de personnages et de } \\
\text { rôles } \\
\begin{array}{l}\text { Recours à un lexique } \\
\text { spécialisé }\end{array}\end{array}$ & $\begin{array}{l}\text { Mais en vérité ce n'était pas un éléphant } \\
\text { mais un chaman, le chaman du village de } \\
\text { Tembo. } \\
\text { «Bienvenu dans notre camp, tu es notre } \\
\text { cornac à tous maintenant» }\end{array}$ \\
\hline Texte enclencheur & $\begin{array}{l}\text { Reprise d'un procédé } \\
\text { syntaxique: } \\
\text { phrases nominales }\end{array}$ & $\begin{array}{l}\text { Tout d'abord, faire attention. } \\
\text { Un éléphant en danger. }\end{array}$ \\
\hline $\begin{array}{l}\text { Texte produit en } \\
\text { première version }\end{array}$ & $\begin{array}{l}\text { Auto reformulations du texte } \\
\text { déjà-produit: } \\
\text { J'entendais les vagues, les } \\
\text { bruits de la mer, et les } \\
\text { oiseaux. }\end{array}$ & $\begin{array}{l}\text { J'entendais la mer, ses vagues et je voyait } \\
\text { tout cet océan. }\end{array}$ \\
\hline Texte & $\begin{array}{l}\text { Reformulation du texte B : } \\
\text { Je me trouvai bientôt en } \\
\text { pleine jungle. }\end{array}$ & $\begin{array}{l}\text { Mais je croyait que je serai seul sans } \\
\text { personne dans la jungle }\end{array}$ \\
\hline
\end{tabular}

Cette élève parvient à tirer profit de toutes les ressources déjà-là : celles acquises, celles fournies par les conditions de l'expérimentation, celles conquises par une première production.

\section{Conclusion : tirer profit du déjà-là et apprendre à le maitriser}

93 Nous concluons que, dans notre protocole, les actes décisionnels des scripteurs sont en partie déterminés par la mise en mémoire d'un déjà-là (substrat dans ses aspects linguistiques et diégétiques) issu de lectures et d'éléments culturels antérieurs, ou rendu disponible par les textes injonctifs (consigne et texte enclencheur) et les textes ressources, et par le souvenir du texte déjà-produit en première version qui impose lui aussi ses choix au texte à venir.

Ces éléments altèrent les degrés de liberté offerts au scripteur. Soit ils s'imposent (par discipline scolaire ou interprétation du contrat didactique en faveur d'une conformité à une norme langagière inférée ?) comme un carcan contraignant à partir duquel l'élève, comme un équilibriste, bâtit difficilement le scénario d'une histoire recomposée sur le fil des emprunts langagiers. Soit ils balisent le déroulement narratif, offrant un espace pour respirer au jeune scripteur qui peut se consacrer à une création plus personnelle puisque les matériaux langagiers captés se révèlent être des points d'appui lors de la textualisation. Nous avons vu aussi, sans pouvoir l'expliquer, que certains apprenants choisissent de s'affranchir de toutes références au corpus fourni. Ce déjà-là peut pourtant 
se constituer en ressort de reformulations, facteurs de remobilisation langagière et de réinvestissement inscrits dans un processus d'apprentissage continu. Toutefois si le substrat récupéré ou fourni lors de la production écrite peut contribuer à la structuration du scénario et à cet épaississement du texte dont parle D. Bucheton (1996), il ne fait pas tout le texte. Le substrat emprunté peut imposer ses contours, mais le puzzle ne s'emboite pas sans force et déchirement : récupérations partielles, aléatoires, intégrations insolites, reformulations tâtonnantes entrainant dans leurs sillages d'autres chocs comme des décrochages énonciatifs. Les opérations de surface, la cohésion et la cohérence du texte font parfois défaut.

C'est tout l'enjeu de la didactique que de proposer des moyens de récupérer et de dompter ce déjà-là pour apprendre à écrire.

\section{BIBLIOGRAPHIE}

BARTHES, R. (1970). S/Z. Paris : Seuil.

BESSONNAT, D. (2000). « Deux ou trois choses que je sais de la réécriture ». Pratiques 105/106, p. 5-22.

BISHOP, M.-F (2006). « Les écritures de soi à l'école primaire : bref historique d'un genre scolaire ». Repères 34, p. 21-40.

- (2007). « L'élève sujet dans les textes prescripteurs de l'école primaire ». Le français aujourd'hui 157, p. 19-29.

BORÉ, C. (2000). « Le brouillon, introuvable objet d'étude ». Pratiques 105/106, p. 23-50.

BORÉ, C. \& CALIL, E. (dirs) (2013). L'école, l'écriture et la création : études françaises et brésiliennes. Louvain-La-Neuve : Academia-L'Harmattan.

BORÉ, C. \& DOQUET-LACOSTE, C. (2004). « La réécriture, questions théoriques ». Le français aujourd'hui 144, p. 9-17.

BORÉ, C. \& HUYNH, J. A. (2004). « Réécritures ». Le français aujourd'hui 144, p. 3-7.

BOUTAN, P. (1996). La langue des Messieurs. Histoire de l'enseignement du français à l'école primaire.

Paris : A. Colin.

BRANCA-ROSOFF, S., \& GARCIA-DEBANC, C. (2007). « L'avant-texte à l'école primaire. Quelques jalons de 1850 à nos jours ». Langue française 155, p. 35-50.

BUChEton, D. (1996). « L'épaississement du texte par la réécriture ». In : David, J. \& Plane, S. L'apprentissage de l'écriture, de l'école au collège. Paris : Presses universitaires de France, p. 159-184.

BUCHETON, D., CHABANNE, J.-C., DECRON, A. \& SEWERYN, B. (2000). « Réécrire ou penser à nouveau son texte? » Pratiques 105/106, p. 203-212.

CAUtela, A., \& Marin, B. (2013). « Emprunts et empreintes textuel(le)s ». Le français aujourd'hui 181, p. 37-45.

CHERVEL, A. (2008). Histoire de l'enseignement du français du XVII au XX siècle. Paris : Retz. 
CRINON, J. (2006). « Lire et écrire la fiction : quelques malentendus ». Repères 33, p. 61-79.

DAUNAY, B. (2004). « Réécriture et paraphrase. Contribution à une histoire des pratiques d'écriture scolaire ». Le français aujourd'hui 144, p. 25-32.

DONAHUE, T. (2007). « Activités de reformulation dans des textes d'étudiants en première année universitaire ». In : Kara, M. (dir.), Usages et analyses de la reformulation. Metz : Université Paul Verlaine, p. 243-269.

DOQUet-lacoste, C., Lumbroso, O. \& TAUVERon, C. (2009). «Écrire avec, sur, de la littérature ».

Repères 40, p. 5-8.

DORMOY, D. (2004). « De la réécriture comme mode d'écriture de textes. Ou apprendre à écrire en réécrivant ». Le français aujourd'hui 144, p. 53-61.

DUBOIS-MARCOIN, D. (1997). Égarements, évanouissements dans les robinsonnades. Cahiers Robinson no 1. Voyages d'enfants : contre la ligne. En ligne : http://lescahiersrobinson.univ-artois.fr/index.php? livre $=1$.

DUFAYS, J.-L., \& KERVYN, B. (2004). « Le rôle des stéréotypes dans l'écriture fictionnelle au Collège ». Didactique de la lecture et de l'écriture littéraires hors série 1, p. 115-121.

FRADET, M.-F. (2006). «Construction de la fiction et interprétation de la consigne dans les écrits de 6e ». Repères 33, p. 123-150.

FRANÇOIS, F. (1990). «Plaisir et structure. L'enfant “créateur" : le texte des adultes, sa reformulation par l'enfant ». Enfances 43, p. 177-186.

FUCHS, C. (1994). Paraphrase et énonciation. Gap : Ophrys.

GENETTE, G. (1982). Palimpsestes : la littérature au second degré. Paris : Seuil.

HALTÉ, A. (2004) «La robinsonnade en littérature de jeunesse contemporaine : genre et valeurs », Skholê hors série 1, p. 21-29.

JEY, M. (2006). «L'étude de fiction : un objet introuvable dans l'école de la République ?» Repères 33 , p. 21-35.

KARA, M. (dir.) (2007). Usages et analyses de la reformulation. Metz : Université Paul Verlaine.

KRISTEVA, J. (1969). Semeiotiké. Recherches pour une sémanalyse. Paris : Seuil.

LE GOFF, F. (2006). Écriture d'invention, réécriture et enseignement de la littérature. Thèse de doctorat en Sciences du langage : Université de Metz.

LECLAIRE-HALTÉ, A. (2000). Les robinsonnades en littérature de jeunesse contemporaine : genre et valeurs. Thèse de doctorat en Sciences du langage : Université de Metz.

LEJEUNE, P. (1995). Signes de vie. Le pacte autobiographique 2. Paris : Seuil.

MARTINOT, C. (2007). «Quand acquisition rime avec reformulation : nécessité d'une réponse linguistique aux phénomènes d'acquisition de la langue maternelle ». In : Kara, M. (dir.) Usages et analyses de la reformulation. Metz : Université Paul Verlaine, p. 179-211.

- (2015). «La reformulation : de la construction du sens à la construction des apprentissages en langue et sur la langue ». Corela HS-18. En ligne : http://corela.revues.org/4034.

Petitjean, A. (1984). « Pastiche et parodie : enjeux théoriques et pédagogiques ». Pratiques 42, p. 3-34. 
PLANE, S. (2000). « Éléments pour un usage didactique sur traitement de texte. Écrire, réécrire et réviser sur ordinateur ». Pratiques 105/106, p. 159-181.

- (2006a). «Singularités et constantes de la production d'écrit. L'écriture comme traitement de contraintes ». In : Laffont-Terranova, J. \& Colin, D. (éds), Didactique de l'écrit. La construction des savoirs et le sujet-écrivant. Namur : Presses Universitaires de Namur, p. 33-54.

- (2006b). « L'écriture de fiction existe-t-elle ?» Repères 33, p. 7-19.

- (à paraître). « Dynamique de l'écriture et processus de resémantisation ». Travaux Linguistiques du Cerlico 26.

PLANE, S., RONDELLI, F., \& VÉNÉRIN, C. (2013). « Variations, fidélité, infidélité : l'écriture et la réécriture de discours rapportés par de jeunes scripteurs ». In : Desoutter, C \& Meilet, C. (dirs), Le discours rapporté : approches linguistiques et perspectives didactiques. Berne : Peter Lang, p. 215-232.

ROSSARI, C. (1994). Les opérations de reformulation: analyse du processus et des marques dans une perspective contrastive français-italien. Berne : Peter Lang.

TAUVERON, C., \& Sève, P. (2005). Vers une écriture littéraire ou comment construire une posture d'auteur à l'école de la GS au CM2. Paris : Hatier.

\section{Littérature primaire}

DEFOE, D. Robinson Crusoé (1977) [1719]. Paris : École des loisirs, p. 36-38, p. 42-43.

GoLDiNG, W. (1978) [1954]. Sa Majesté les Mouches. Trad. de l'anglais par L. Tranec-Dubled. Paris : Gallimard, p. 78-79.

MORPURGO, M. (2000). Le royaume de Kensuké. Paris : Gallimard, p. 43-44, p. 47-49, p. 50-51.

STEIG, W. L'île d'Abel (2003) [1982]. Trad. de l'anglais par H. Robillot. Paris : École des loisirs, p. $56-58$

TOURNIER, M. (2011) [1971]. Vendredi ou la vie sauvage. Paris : Flammarion, p. 43-44.

\section{NOTES}

1. Institut des textes et manuscrits modernes - CNRS.

2. Nous renvoyons aux travaux de C. Fuchs (1994, p. 7) et de B. Daunay (2004) qui relient la notion à la pratique de l'explication de texte ou à l'écriture d'invention au lycée comme activité de « réécriture de textes".

3. C. Fuchs (1994, p. 7) appelle paraphrase imitative (ou reformulation (à visée) imitative) celle «qui se centre, elle, sur la production du texte-cible T' dont elle cherche à construire les formes d'expression à partir du sens du texte-source $\mathrm{T}$ ».

4. C. Rossari (1994) distingue les reformulations paraphrastiques, qui instaurent une équivalence avec la première formulation, des reformulations non paraphrastiques, lesquelles opèrent un changement de perspective énonciative.

5. Consigne orale: «Vous aurez à votre disposition quatre textes d'écrivains que vous pourrez lire puis utiliser... ». Consigne écrite au tableau : « Vous avez des textes à votre disposition. »

6. Test statistique de Kolmogorov-Smirnov.

7. Test de Wilcoxon et test de Mann-Whitney. 
8. En effet, les différences observées (G1V 1> G1V2; G2V1 > G2V2) ne sont pas significatives (valeurs de « $p$ » très éloignées du seuil de $5 \%$ ). On ne peut pas exclure que cette absence de significativité ne soit pas due à un manque de puissance (corpus de trop petite taille). Néanmoins l'écart important entre les valeurs de " $\mathrm{p}$ » et le seuil de 0,05 n'est pas en faveur de cette hypothèse, et l'on peut inférer que, dans cette étude, la répétition ne modifie pas la longueur du texte produit.

9. $p=0,04$ : en première version, les suites de récits écrits sont plus longues que les créations de récits. Selon les comparaisons effectuées, ce résultat n'est pas dû au hasard.

\section{RÉSUMÉS}

Cet article porte sur la manière dont des scripteurs débutants emploient et réinvestissent des matériaux linguistiques issus à la fois d'un texte qu'ils ont eux-mêmes produit et d'un corpus de textes littéraires qui leur a été fourni. Il s'intéresse aux modes de récupération de ces matériaux et à la façon dont ceux-ci s'intègrent au texte nouvellement produit. Les observations menées pour identifier et caractériser ces traitements ont porté sur la production de récits ou de suites de récits relevant du genre de la robinsonnade par 48 élèves de fin d'école primaire au cours de deux phrases d'écriture. Ces élèves ont eu à leur disposition un corpus d'extraits littéraires de robinsonnades lors de la deuxième phase d'écriture. L'analyse des données mène vers deux constats. D'une part, on note que la récupération des éléments provenant des textes sources s'effectue selon deux formes différentes de fidélité à ces textes, l'une privilégiant le prélèvement d'éléments, l'autre la restitution sémantique. D'autre part, on observe deux tendances contrastées lors de la réécriture, l'une consistant à maintenir le système déployé en première version et à accorder la prépondérance aux matériaux issus de cette première phase, l'autre dans laquelle les actes décisionnels sont largement déterminés par le substrat littéraire fourni. Ces tendances présentent elles-mêmes des variantes qui sont marquées par l'influence du déjà-là culturel dont dispose l'apprenant. Ces constats fournissent des éléments pour caractériser des modes d'écriture et devraient ouvrir vers des pistes didactiques.

This article examines how novice scriptwriters use and reinvest linguistic elements from both a text that they had previously written themselves and a corpus of literary texts that had been provided to them. In particular, this article focuses on how such elements are re-exploited to fit into a newly generated text. The observations carried out to identify and characterise these processes derive from the production of narratives and of successions of stories of the "robinsonade" genre by 48 pupils at the end of primary school following a two-phase process. These pupils were provided with a body of "robinsonade" extracts during the second writing phase. The data analysis leads to two conclusions. First, the recycling of elements from the source texts stems from two different forms of loyalty towards the said texts: one favouring the removal of elements, the other favouring semantic restitution. Secondly, two contrasting trends are observed when it comes to rewriting. The first one consists in maintaining the system originally used to produce the first version and to favour elements used during this first phase. In the second one, decision-making is largely determined by the literary substrate that is provided. Variants exist amongst these tendencies and they are influenced by the "alreadythere" culture of the one who learns. The above findings provide material to characterise ways of writing and should help open new didactic approaches. 
INDEX

Keywords : "already-there", school writing, rewriting, literary loan, reformulation

Mots-clés : déjà-là, écriture scolaire, réécriture, emprunt, reformulation

\section{AUTEUR}

\section{KATHY SIMILOWSKI}

Université Paris-Sorbonne, STIH, EA 4509, F-75005 